\section{Height-Reduced Meander Zigzag Monopoles with Broad-Band Characteristics}

\author{
JMMMY L. WONG, MEMBER, IEEE, AND HOWARD E. KING, \\ SENIOR MEMBER, IEEE
}

\begin{abstract}
The characteristics of a meander dual zigzag wire monopole antenna are described. The antenna provides low height and broad-band performance. Measured voltage standing-wave ratio (VSWR) and pattern characteristics are compared with a conventional, straight-wire monopole over a 3:1 bandwidth.
\end{abstract}

\section{INTRODUCTION}

In many practical situations it is desirable to reduce the size (height) of the antenna for various reasons such as compactness and cost. For the present study, the antenna design is for use on a spacecraft whose dimension is $<\lambda / 3$ at the lowest operating frequency, and the antenna is constrained to fit within a small allotted space to avoid complex deployment. In addition, the antenna system is required to provide operation over two broad VHF bands within a 3:1 high-to-low frequency ratio -50 to $80 \mathrm{MHz}$ and 110 to 150 $\mathrm{MHz}$. The frequency requirements can be satisfied with either a single, dual-band antenna or two separate broad-band antennas.

A great deal of research has been devoted to the development of wide-band, electrically small antennas; however, none of the sizereduced wire-type antennas has been shown to possess wide bandwidth impedance characteristics [1]-[5]. This communication describes a new type of monopole antenna whose height is approximately half and whose bandwidth is nearly twice that of a conventional $\lambda / 4$ straight wire monopole. It is further shown that, with a proper choice of design parameters, the antenna can be operated over two widely separated bands with little or no change in pattern performance relative to a tuned $\lambda / 4$ monopole. The experimental measurements reported herein were performed in the scale frequency range of $250 \mathrm{MHz}$ to $750 \mathrm{MHz}$.

\section{DESIGN DESCRIPTION}

The antenna consists of a driven element fed from a coaxial line and one or more closely spaced parasitic elements (open sleeves) shorted to a ground plane. The basic elements are constructed of two identical meander wires in a zigzag configuration and fed from a common feed point (see Figs. 1 and 2). For this reason, the antenna may be described as a meander dual zigzag monopole. The dual zigzag configuration was chosen to provide symmetry and reduce cross-polarization distortion. The geometry and size of the sleeve(s) may be varied to obtain the desired bandwidth characteristics.

\section{RF CHARACTERISTICS}

Fig. 1 shows the voltage standing-wave ratio (VSWR) response of a typical meander dual zigzag monopole with one open sleeve. Both the driven element and the open sleeve were constructed with no. 12 ( 0.081 in) wires. This meander monopole was intended for use as a single broad-band antenna in the 250 to $400 \mathrm{MHz}$ range. The bandwidth performance of a 7.06 in long, straight-wire monopole with a resonant frequency of $\sim 400 \mathrm{MHz}$ is also shown for comparison. The overall height of the meander monopole is 5.5 in as

Manuscript received October 25, 1985; revised November 19, 1985. This work was supported by the U.S. Air Force Space Division under Contract F04701-84-C-0085.

The authors are with the Electronics Research Laboratory, The Aerospace Corporation, El Segundo, CA 90245.

IEEE Log Number 8407327.

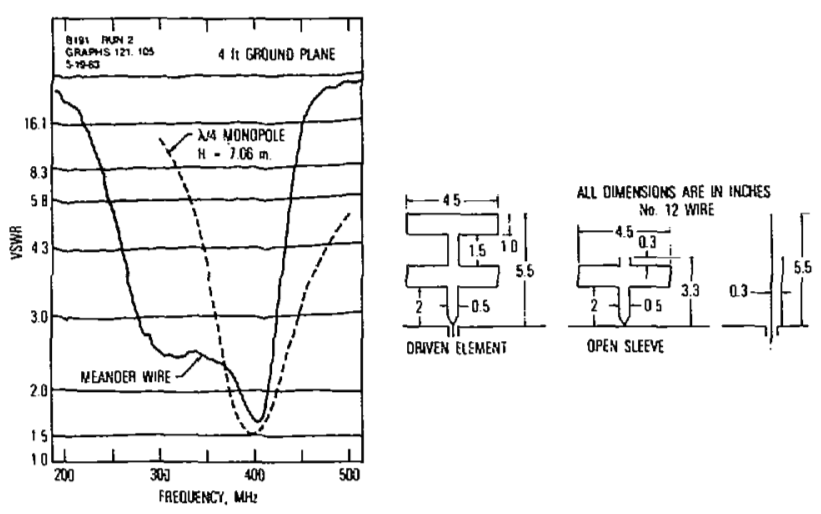

Fig. 1. VSWR of typical meander monopole with one open sleeve.

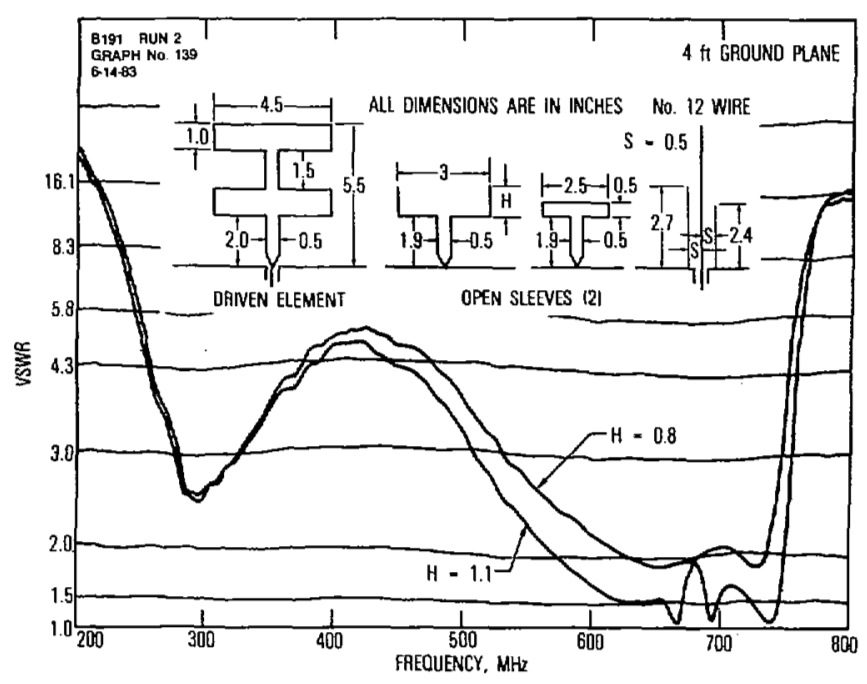

Fig. 2. VSWR of dual-band meander monopole with two open sleeves,

can be seen from Fig. 1. Note that a tuned monopole at $250 \mathrm{MHz}$ would require a wire length of $\sim 11 \mathrm{in}$.

Fig. 2 shows another meander monopole with two open sleeves. In this case, the driven element is the same as Fig. 1, and the sleeve parameters and spacing were adjusted to provide operation over two widely separated bands within the 250 to $750 \mathrm{MHz}$ frequency range. The VSWR performance depends on the bandwidth requirement in each band, and it is $<5.5: 1$ over the measurement frequency range. For certain communication systems, such as the one being considered for a receiving application, where antenna gain is not a critical factor, the VSWR performance of Fig. 2 may be adequate over the entire 3:1 frequency range. Pattern measurements were made from 250 to 750 $\mathrm{MHz}$ with the meander monopole of Fig. 2 mounted on a $4 \mathrm{ft}$ diameter ground plane. Typical patterns measured in the plane of the meander at $250,325,400,550,625$, and $750 \mathrm{MHz}$ are shown in Fig. 3. For comparison, the pattern of a tuned $\lambda / 4$ straight monopole at each of the test frequencies is also shown. Patterns measured in the plane perpendicular to the meander are similar to those shown in Fig. 3. Although the pattern of a monopole depends on the ground plane size and wavelength, it can be seen that the pattern of the meander monopole resembles very closely that of a $\lambda / 4$ monopole at each of the test frequencies. The basic $\lambda / 4$ monopole pattern is retained over a 3:1 band. It should be mentioned that a straight wire monopole with a length of $\lambda / 4$ at the lowest operating frequency is not capable of preserving the basic pattern when the bandwidth exceeds an octave. 

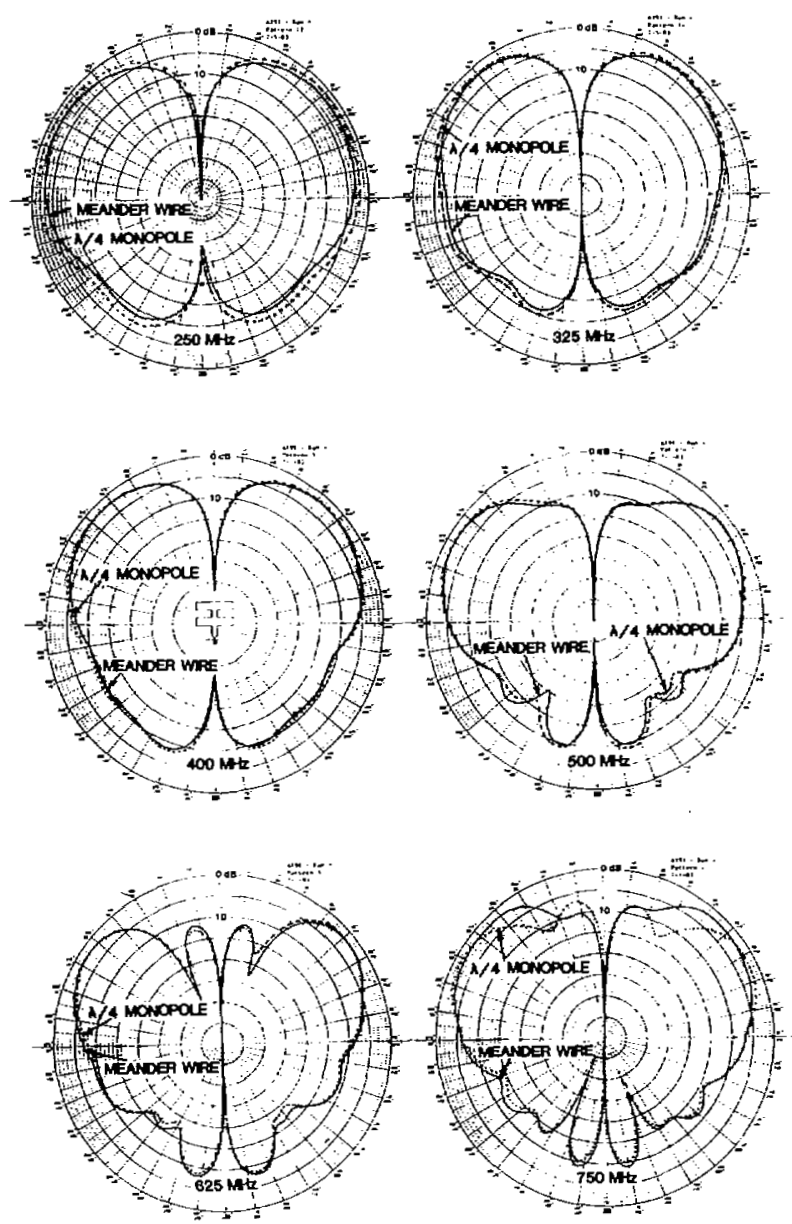

Fig. 3. Measured patterns of meander monopole with 250 to $750 \mathrm{MHz}$.

\section{CONCLUSION}

The broad-band characteristics of a meander dual zigzag wire monopole with a height reduction of $\sim 50$ percent have been demonstrated. By appropriate choice of sleeve parameters and spacing(s), the meander monopole can be tuned for single or dualband operation over a wide frequency range. When the meander monopole is operated in conjunction with a ground plane, the basic $\lambda /$ 4 straight monopole pattern characteristics are preserved over a $3: 1$ frequency range.

\section{REFERENCES}

[1] E. C. Jordan, "Antenna practice and design," in Electromagnetic Waves and Radiating Systems. Englewood Cliffs, NJ: PrenticeHall, 1950 , ch. 14 , p. 512 .

[2] M. Eovine, "Helical monopole HF antenna," Chu Associates, Littleton, MA, Final Rep., Oct. 15, 1962. DDC no. AD 288270.

[3] T. Kitsuregawa, Y. Takeichi, and M. Mizusawa, "A one eighth wave broadband folded unipole antenna," Electromagn. Theory and Antennas Symp. Proc., Copenhagen, Denmark, June 1962, vol. 6, pt. 2, New York: Pergamon, E. C. Jordan, Ed.

[4] W. A. Edson, "Broadband trapped multiple-wire antenna," in 1981 IEEE Antennas Propagat. Soc. Symp. Dig., pp. 586-589.

[5] J. Rashed and C. T. Tai, "A new class of wire antennas," in 1982 IEEE Antennas Propagat., Soc. Symp. Dig., vol. 2, pp. 564-567.

\section{Slant Path Absorption Correction for Low Elevation Angles}

EDWARD E. ALTSHULER, FELLOW, IEEE

\begin{abstract}
Slant path attenuation at millimeter wavelengths due to the gases oxygen and water vapor is often estimated by multiplying the zenith attenuation by the cosecant of the elevation angle. This flat-earth approximation is not valid at low elevation angles. The error produced by this approximation is examined. It is assumed that the actual atmosphere can be represented by 2 uniform surface layer with an effective height such that the attenuation through the layer is equal to that through the actual atmosphere. An expression for calculating this effective height is provided and with the effective height specified, the error produced by the approximation is determined. The percent error is then plotted as a function of effective height for a set of elevation angles above $2^{\circ}$.
\end{abstract}

\section{INTRODUCTION}

Slant path absorption at millimeter wavelengths due to the atmospheric gases oxygen and water vapor, can be measured using the sun as a source [1]. The usual procedure is to make a set of extinction measurements as a function of zenith angle and then infer the zenith attenuation from these data. The slant path attenuation is then calculated by multiplying the zenith attenuation by the cosecant of the elevation angle.

$$
A(\theta)=A\left(\theta=90^{\circ}\right) \csc \theta .
$$

However, this flat-earth approximation is not valid at low elevation angles. In this communication, the error introduced by this approximation is examined.

\section{Estimation of Slant Path attenuation}

The flat-earth approximation is often used for elevation angles above $8^{\circ}$. In order to calculate the attenuation at lower angles, the height profiles of the absorption coefficients of oxygen and water vapor must be known. Since this information is not usually available, the concept of an effective height is used. The effective height is the height of a surface layer of atmosphere consisting of a uniform distribution of oxygen or water vapor that produces the same total absorption as is produced by the actual atmosphere. If the absorption coefficient decreases exponentially, then the effective height is equivalent to the more commonly referred to scale height, the height at which the absorption coefficient decreases to $1 / e$ of its value at the surface. If the height profile of the absorption coefficient is not known, then the effective height is the ratio of the zenith absorption to the surface absorption coefficient.

Assuming that the absorbing atmosphere can be represented by an effective height, the distance of the slant path through the layer surrounding the curved earth is [2]

$$
d_{c}=\left[\left(a+h_{e}\right)^{2}-a^{2} \cos ^{2} \theta\right]^{1 / 2}-a \sin \theta
$$

Manuscript received June 28, 1985.

The author is with the Electromagnetic Sciences Division, Rome Air Development Center, Hanscom AFB, MA 01731.

IEEE Log Number 8407333. 